\title{
Cosmological density perturbations in modified gravity theories
}

\author{
A. de la Cruz-Dombriz, A. Dobado and A. L. Maroto \\ Departamento de Fisica Teórica I, Universidad Complutense de Madrid, 28040 Madrid, SPAIN
}

\begin{abstract}
In the context of $f(R)$ theories of gravity, we study the cosmological evolution of scalar perturbations by using a completely general procedure. We find that the exact fourth-order differential equation for the matter density perturbations in the longitudinal gauge, reduces to a second-order equation for sub-Hubble modes. This simplification is compared with the standard (quasi-static) equation used in the literature. We show that for general $f(R)$ functions the quasistatic approximation is not justified. However for those $f(R)$ adequately describing the present phase of accelerated expansion and satisfying local gravity tests, it does give a correct description for the evolution of perturbations.
\end{abstract}

Keywords: Cosmology, Modified theories of gravity

PACS: 98.80.-k, 04.50.Kd, 95.36.+ $\mathrm{x}$

\section{INTRODUCTION}

The present phase of accelerated expansion of the universe [1] poses one of the most important problems of modern cosmology. It is well known that ordinary Einstein's equations in either a matter or radiation dominated universe give rise to decelerated periods of expansion. In order to have acceleration, the total energy-momentum tensor appearing on the right hand side of the equations should be dominated at late times by a hypothetical negative pressure fluid usually called dark energy [2].

However, there are other possibilities to generate a period of acceleration where Einstein's gravity itself is modified. In one of such possibilities, new functions of the scalar curvature $(f(R)$ terms) are included in the gravitational action. Although such theories are able to describe the accelerated expansion on cosmological scales correctly, they typically give rise to strong effects on smaller scales, but nevertheless, viable models can be constructed imposing strong constraints over $f(R)$ [3]: $f_{R R}>0$ for high curvatures, $1+f_{R}>0$ for all $R, f_{R}<0$ to ensure that ordinary General Relativity behaviour is recovered at early times and $\left|f_{R}\right| \ll 1$ at recent epochs to ensure that local gravity tests hold. $f_{R}$ and $f_{R R}$ mean first and second derivative respectively of $f(R)$ with respect to the argument $R$.

The important question that arises is therefore how to discriminate dark energy models from modified gravities using observations. It is known that by choosing particular $f(R)$ functions, one can mimic any expansion history, and in particular that of $\Lambda \mathrm{CDM}$. Accordingly, the exclusive use of observations from SNIa [1], baryon acoustic oscillations [4] or CMB shift factor [5], based on different distance measurements which are sensitive only to the expansion history, cannot settle the question of the nature of dark energy [6]. 
However, there exists a different type of observations which are sensitive, not only to the expansion history, but also to the evolution of matter density perturbations. The fact that the evolution of perturbations depends on the specific gravity model, i.e. it differs in general from that of Einstein's gravity even though the background evolution is the same, means that this kind of observations will help distinguishing between different models for acceleration.

In this work [7] we find the exact equation for the evolution of matter density perturbations for arbitrary $f(R)$ theories. Such problem had been previously considered in the literature [8] and approximated equations have been widely used based on the so called quasi-static approximation in which all the time derivative terms for the gravitational potentials are discarded, and only those including density perturbations are kept. From our exact result, we will be able to determine under which conditions such an approximation can be justified.

\section{DENSITY PERTURBATIONS EVOLUTION IN $f(R)$ THEORIES}

Let us consider the modified gravitational action:

$$
S=\frac{1}{16 \pi G} \int d^{4} x \sqrt{-g}(R+f(R))
$$

The corresponding equations of motion for that action are modified with respect to their usual General Relativity counterpartners. In our research we use a flat Robertson-Walker perturbed metric in the longitudinal gauge

$$
d s^{2}=a^{2}(\eta)\left[(1+2 \Phi) d \eta^{2}-(1-2 \Psi)\left(d r^{2}+r^{2} d \Omega_{2}^{2}\right)\right]
$$

where $\Phi \equiv \Phi(\eta, \vec{x})$ and $\Psi \equiv \Psi(\eta, \vec{x})$ are the scalar perturbations. Using this perturbed metric and a perturbed energy-momentum tensor for dust matter perfect fluid, the first order perturbed equations may be written for a general $f(R)$ function assuming that background equations hold. A relevant point of these theories is that potentials $\Phi$ and $\Psi$ are not equal provided $f_{R R} \neq 0$. From Einstein's equations, it is possible to derive a fourth order differential equation for matter density perturbation $\delta \equiv \delta \rho / \rho_{0}$ - with $\rho_{0}$ unperturbed density and $\delta \rho$ density constrast with respect to the background -alone. The process can be found in [7]. The resulting equation can be written as follows:

$$
\beta_{4, f} \delta^{i v}+\beta_{3, f} \delta^{\prime \prime \prime}+\left(\alpha_{2}+\beta_{2, f}\right) \delta^{\prime \prime}+\left(\alpha_{1}+\beta_{1, f}\right) \delta^{\prime}+\left(\alpha_{0}+\beta_{0, f}\right) \delta=0
$$

where the coefficients $\beta_{i, f}(i=1, \ldots, 4)$ involve terms with $f_{R}^{\prime}$ and $f_{R}^{\prime \prime}$. Equivalently, $\alpha_{i}$ ( $i=0,1,2$ ) contain terms coming from the linear part (Einstein-Hilbert) of $f(R)$ in $R$ (background curvature). Parameter $\varepsilon \equiv \mathscr{H} / k \equiv a^{\prime} /(a k)$, where' denotes derivative with respect to conformal time $\eta$, will be useful to perform a perturbative expansion of the previous coefficients $\alpha$ 's and $\beta$ 's in the sub-Hubble limit. We also define the following dimensionless parameters: $\kappa_{i} \equiv \mathscr{H}^{(i)} / \mathscr{H}^{i+1}$ and $f_{i} \equiv f_{R}^{(j)} /\left(\mathscr{H}^{j} f_{R}\right)(i, j=1,2,3)$. 


\section{SUB-HUBBLE MODES AND THE QUASI-STATIC LIMIT}

We are interested in the possible effects on the growth of density perturbations once they enter the Hubble radius in the matter dominated era. In the sub-Hubble limit $\varepsilon \ll 1$, it can be seen that the $\beta_{4, f}$ and $\beta_{3, f}$ coefficients are supressed by $\varepsilon^{2}$ with respect to $\beta_{2, f}$, $\beta_{1, f}$ and $\beta_{0, f}$, i.e., in this limit the equation for perturbations reduces to the following second order expression:

$$
\delta^{\prime \prime}+\mathscr{H} \delta^{\prime}+\frac{\left(1+f_{R}\right)^{5} \mathscr{H}^{2}\left(-1+\kappa_{1}\right)\left(2 \kappa_{1}-\kappa_{2}\right)-\frac{16}{a^{8}} f_{R R}^{4}\left(\kappa_{2}-2\right) k^{8} 8 \pi G \rho_{0} a^{2}}{\left(1+f_{R}\right)^{5}\left(-1+\kappa_{1}\right)+\frac{24}{a^{8}} f_{R R}^{4}\left(1+f_{R}\right)\left(\kappa_{2}-2\right) k^{8}} \delta=0
$$

where we have taken only the leading terms in the $\varepsilon$ expansion for the $\alpha$ and $\beta$ coefficients. This expression can be compared with that usually considered in literature, obtained after performing strong simplifications in the perturbed equations by neglecting time derivatives of $\Phi$ and $\Psi$ potentials. Thus in [9] they obtain:

$$
\delta^{\prime \prime}+\mathscr{H} \delta^{\prime}-\frac{1+4 \frac{k^{2}}{a^{2}} \frac{f_{R R}}{1+f_{R}}}{1+3 \frac{k^{2}}{a^{2}} \frac{f_{R R}}{1+f_{R}}} \frac{4 \pi G \rho_{0} a^{2} \delta}{1+f_{R}}=0
$$

We will now restrict ourselves to models satisfying all the viability conditions mentioned in the introduction, including $\left|f_{R}\right| \ll 1$.

Note that the quasi-static expression (5) is only recovered in the matter era (i.e. for $\mathscr{H}=2 / \eta$ ) or for a pure $\Lambda \mathrm{CDM}$ evolution for the background dynamics. Nevertheless in the limit $\left|f_{R}\right| \ll 1$ it can be proven using the background equations of motion that $1+\kappa_{1}-\kappa_{2} \approx 0$ and therefore $2 \kappa_{1}-\kappa_{2} \approx-2+\kappa_{2} \approx-1+\kappa_{1}$ what is nothing but the fact that for viable models the background evolution resembles that of $\Lambda \mathrm{CDM}$. This this fact allows to simplify expression (3) to approximately become (5).

In other words, although for general $f(R)$ functions the quasi-static approximation is not justified, for those viable functions describing the present phase of accelerated expansion and satisfying local gravity tests, it gives a correct description for the evolution of perturbations.

In order to check our results we proposed two particular $f(R)$ models to obtain solutions both using (4) and (5). As commented before, for viable models the background evolution resemble that of $\Lambda \mathrm{CDM}$ at low redshifts and that of a matter dominated universe at high redshifts. Nevertheless the $f(R)$ contribution gives the dominant contribution for small curvatures and therefore it may explain the cosmological acceleration.

The two studied models are of the form $f(R)=c_{1} R^{p}$ (where units $H_{0}^{2}$ will be considered). According to the results presented in [10], models of this type include both matter dominated and late-time accelerated universe provided the parameters satisfy $c_{1}<0$ and $0<p<1$.

The first model (left figure) will have parameters $c_{1}=-4$ and $p=0.63$ and it is not accomplishing condition $\left|f_{R}\right| \ll 1$. Second model (right figure) will have $c_{1}=-4.3$ and $p=0.01$ and it does accomplish condition $\left|f_{R}\right| \ll 1$. For each model, we have compared our result with the standard $\Lambda \mathrm{CDM}$ and the quasi-static approximation (5). We saw that for first model quasi-static and correct approximation do not give the 
same evolution but for the second one the quasi-static approximation gives a correct description for the evolution.
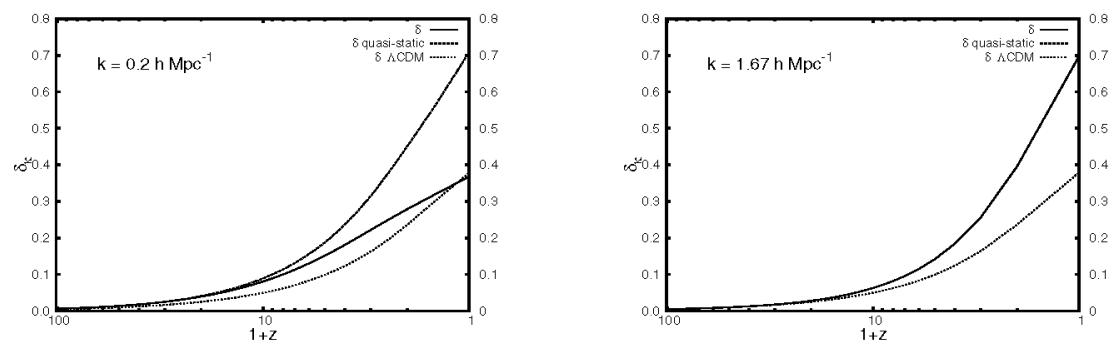

Left figure: $\delta_{k}$ with $k=0.2 \mathrm{hMpc}^{-1}$ for first model and $\Lambda \mathrm{CDM}$. Both, standard quasi-static evolution and equation (4) have been plotted and they clearly differ. ; Right figure: $\delta_{k}$ with $k=1.67 \mathrm{hMpc}^{-1}$ for second model and $\Lambda C D M$. The quasi-static evolution is indistinguishable from that coming from (4), but both diverge from $\Lambda$ CDM behaviour as $z$ decreases.

\section{CONCLUSIONS}

We have shown that for sub-Hubble modes, the differential equation for the evolution of density perturbation reduces to a second order equation and compared this result with that obtained within quasi-static approximation used in the literature and found that for arbitrary $f(R)$ functions, such an approximation is not justified.

However for theories with $\left|f_{R}\right| \ll 1$ today, perturbative calculation for sub-Hubble modes requires to take into account, not only the first terms, but also higher-order terms in $\varepsilon=\mathscr{H} / k$. In that case, the resummation of such terms modifies the equation which can be seen to be equivalent to the quasi-static case, but only if the universe expands as in a matter dominated phase or in a $\Lambda$ CDM model. Finally, the fact that for models with $\left|f_{R}\right| \ll 1$ the background behaves today precisely as that of $\Lambda$ CDM makes the quasistatic approximation correct in those cases.

Acknowledgements: We would like to thank J. A. R. Cembranos and J. Beltrán.This work has been supported by the DGICYT (Spain) under projects FPA 2004-02602 and 2005-02327, CAM/UCM 910309 and by UCM-Santander PR34/07-15875.

\section{REFERENCES}

1. S. Perlmutter et al. [Supernova Cosmology Project Collaboration], Astrophys. J. 517, 565, (1999).

2. E. J. Copeland, M. Sami and S. Tsujikawa, Int. J. Mod. Phys. D, 15, 1753 (2006).

3. L. Pogosian and A. Silvestri, Phys. Rev. D 77 023503, (2008).

4. D. J. Eisenstein et al. Astrophys. J. 633: 560-574, (2005).

5. D. N. Spergel et al. [WMAP Collaboration], Astrophys. J. Suppl. 170 377, (2007).

6. E. Linder. Phys.Rev. D $72: 043529,(2005)$.

7. A. de la Cruz-Dombriz, A. Dobado and A. L. Maroto, Phys. Rev. D 77123515 (2008).

8. S. M. Carroll et al., New J. Phys. 8323, (2006) ; P. Zhang, Phys. Rev. D 73 123504, (2006).

9. S. Tsujikawa, Phys. Rev. D 76023514 , (2007).

10. L. Amendola, R. Gannouji, D. Polarski and S. Tsujikawa, Phys. Rev. D 75083504 (2007). 
Copyright of AIP Conference Proceedings is the property of American Institute of Physics and its content may not be copied or emailed to multiple sites or posted to a listserv without the copyright holder's express written permission. However, users may print, download, or email articles for individual use. 